\title{
Quantum effects due to a moving Dirichlet point
}

\author{
C. D. Fosco, ${ }^{1}$ D. R. Junior, ${ }^{2}$ and L. E. Oxman ${ }^{2}$ \\ ${ }^{1}$ Centro Atómico Bariloche and Instituto Balseiro, Comisión Nacional de Energía Atómica, \\ R8402AGP Bariloche, Argentina \\ ${ }^{2}$ Instituto de Física, Universidade Federal Fluminense, Campus da Praia Vermelha, \\ Niterói, RJ 24210-340, Brazil
}

(Received 28 October 2019; accepted 4 March 2020; published 24 March 2020)

\begin{abstract}
We study quantum effects induced by a pointlike object that imposes Dirichlet boundary conditions along its worldline, on a real scalar field $\varphi$ in 1,2, and 3 spatial dimensions. The boundary conditions result from the strong coupling limit of a term quadratic in the field and localized on the particle's trajectory. We discuss the renormalization issues that appear and evaluate the effective action. Special attention is paid to the case of two spatial dimensions where the coupling constant is adimensional.
\end{abstract}

DOI: 10.1103/PhysRevD.101.065014

\section{INTRODUCTION}

Quantum field theory predicts that an open system is capable of evolving, from the vacuum, to a state characterized by a nonvanishing number of (real) quanta [1]. That is indeed the case, among other related phenomena, of the dynamical Casimir effect (DCE), one of the most studied manifestations of quantum dissipation [2]. The DCE consists of the emission of real quanta when a field is subjected to time-dependent boundary conditions, an example being the presence of one or more moving mirrors, namely, of objects imposing nontrivial boundary conditions on the field. In the usual understanding of the term, a boundary condition acts on a region having codimension one, i.e., which is determined by a single equation. It is worth noting that, in the context of the DCE for a real scalar field, which we consider here, different kinds of boundary conditions, besides the "perfect" ones (Dirichlet and Neumann), have also been studied. Those "imperfect" conditions describe mirrors which have more realistic responses to the action of the field's modes. Among that kind of condition, a relatively simple one amounts to Dirichlet-like boundary conditions: they result from the addition to the action of a term localized on the space-time region which is swept by the mirror during the course of time. When the strength of that term tends to infinity, one gets Dirichlet conditions on the region on which the term is localized. It is our concern in this paper to study the DCE, for the case of a real scalar field $\varphi$ in $d+1$ dimensions

Published by the American Physical Society under the terms of the Creative Commons Attribution 4.0 International license. Further distribution of this work must maintain attribution to the author(s) and the published article's title, journal citation, and DOI. Funded by SCOAP ${ }^{3}$. $(d=1,2,3)$, coupled to pointlike objects which implement precisely that kind of Dirichlet-like boundary conditions. In other words, we shall add to the scalar field Lagrangian a term proportional to a $\delta$ function of the (time-dependent) position of the particle, and to the square of $\varphi$. The strength of the term is determined by a coupling constant which, by taking the appropriate limit, will be used to impose Dirichlet boundary conditions.

We shall follow our previous work for scalar and spinorial vacuum fields $[3,4]$ in which we used the particularly convenient functional approach proposed by Golestanian and Kardar [5]. The approach is based on the use of auxiliary fields to deal with the role of the mirrors, on the calculation of the functional integral for the in-out effective action. An important feature of the systems that we consider here is the following: except for $d=1 \mathrm{a}$ curve, like the particle's worldline, has codimension bigger than 1; this fact results in qualitatively different UV properties during the calculation of the effective action. Indeed, the UV problems which will arise here are rather similar to the ones corresponding to Dirac $\delta$ potentials in 2 and 3 dimensions, a system which has been extensively studied by following many different approaches and frameworks (see, for example, [6-9]). Note that the classical, static Casimir effect for small objects is one of the problems considered in [10], by using a multipole expansion. What we have in mind here is the evaluation of the dynamical and quantum version of that kind of object.

In this paper, we shall first review the $d=1$ case, as a previous step to dealing with $d=2$, and $d=3$. The main distinction between $d=2$ or $d=3$ and $d=1$ are, as we shall see, due to the different UV properties induced by the coupling between the particle and the field. Indeed, the usual renormalization which is required to make sense of a $\delta$-like potential in quantum mechanics in two and three 
spatial dimensions, also manifests itself here; moreover, the resulting divergences can be cured by applying a similar procedure.

The $d=2$ case will also be relevant for future developments regarding the quantum properties of center vortices, and support the construction of phenomenological ensembles for these magnetic defects. They are topological variables that are believed to capture the infrared behavior in Yang-Mills (YM) theories. For a given realization, the calculation of the effective action involves regularity field conditions on worldlines and world surfaces in three and four Euclidean dimensions, respectively, which are problems of codimension 2. The effective action for a single center vortex without curvature was analyzed in Refs. [1115]. This may involve singular spectral problems with different gyromagnetic ratios and regularity conditions on the YM off-diagonal sector, which depend on how the fluctuations are parametrized.

The structure of this paper is as follows: In Sec. II we introduce the kind of system that we study, as well as some general expressions for its effective action in the small departure limit, in the path integral framework. Then, in Sec. III we evaluate the effective action for the massless field, by considering a perturbative expansion in powers of the departure of the worldline from the one of a static particle, assuming the mirror moves nonrelativistically, for $d=1$ and $d=3$. Because of its particularities, related to scale invariance of the coupling between field and mirror, the $d=2$ case is considered separately in Sec. IV. Finally, in Sec. V we present our conclusions.

\section{THE SYSTEM}

The system that we shall deal with throughout this paper consists of a real scalar field $\varphi$ in $d+1$ dimensions, with $d=1,2$ or 3 , coupled to pointlike objects which are meant to implement Dirichlet-like conditions.

For an object imposing Dirichlet conditions, the effective action will be denoted by $\Gamma(\mathcal{C})$, since it is a functional of the worldline $\mathcal{C}$. In a functional integral approach, and using Euclidean conventions, ${ }^{1}$

$$
e^{-\Gamma(\mathcal{C})}=\frac{\mathcal{Z}(\mathcal{C})}{\mathcal{Z}_{0}}
$$

where $\mathcal{Z}(\mathcal{C})\left(\mathcal{Z}_{0}\right)$ denotes the Euclidean vacuum transition amplitude corresponding to the scalar field in the presence (absence) of the particle.

$\mathcal{Z}(\mathcal{C})$ and $\mathcal{Z}_{0}$ are given, explicitly, by

$$
\mathcal{Z}(\mathcal{C})=\int \mathcal{D} \varphi \delta_{\mathcal{C}}(\varphi) e^{-\mathcal{S}_{0}(\varphi)}, \quad \mathcal{Z}_{0}=\int \mathcal{D} \varphi e^{-\mathcal{S}_{0}(\varphi)},
$$

\footnotetext{
${ }^{1} \mathrm{~A}$ Wick rotation back to real time will be performed afterwards when dealing with the calculation of its imaginary part.
}

where $\mathcal{S}_{0}$ is the action which describes the free propagation of the field, and a functional $\delta$ function has been introduced to account for the Dirichlet conditions; namely, the vanishing of the field at the position of the particle. The former is given by

$$
\mathcal{S}_{0}(\varphi)=\frac{1}{2} \int_{x}\left(\partial_{\mu} \varphi(x) \partial_{\mu} \varphi(x)+m^{2} \varphi^{2}(x)\right),
$$

where we have introduced a shorthand notation for the integration, in this case over all of the spacetime coordinates $x=\left(x_{0}, x_{1}, \ldots, x_{d}\right)$. Namely, in the case above, $\int_{x} \equiv \int d^{d+1} x$. Greek indices will be assumed to run over the values $0,1, \ldots, d$, and space-time is endowed with the Euclidean metric: $g_{\mu \nu}=\delta_{\mu \nu}$.

The functional $\delta_{\mathcal{C}}(\varphi)$ should select, among the configurations appearing in the functional integration measure, just the $\varphi$-field configurations which satisfy Dirichlet boundary conditions on $\mathcal{C}$. As already advanced, those conditions will be reached as the limit of a local term, namely: we add to the free action a term, quadratic in $\varphi$ and localized on $\mathcal{C}$, with a strength $\lambda$ which, when $\lambda \rightarrow \infty$, imposes Dirichlet boundary conditions:

$$
\Gamma(\mathcal{C})=\lim _{\lambda \rightarrow \infty} \Gamma_{\lambda}(\mathcal{C}), \quad e^{-\Gamma_{\lambda}(\mathcal{C})}=\mathcal{Z}_{\lambda}(\mathcal{C}) / \mathcal{Z}_{0}
$$

where

$\mathcal{Z}_{\lambda}(\mathcal{C})=\int \mathcal{D} \varphi \exp \left\{-\mathcal{S}_{0}(\varphi)-\frac{\lambda}{2} \int_{\tau} \sqrt{g(\tau)}[\varphi(y(\tau))]^{2}\right\}$

where we have assumed that $\tau \rightarrow y_{\mu}(\tau)(\mu=0,1, \ldots, d)$ is a parametrization of $\mathcal{C}$, and $g(\tau) \equiv \dot{y}_{\mu}(\tau) \dot{y}_{\mu}(\tau)$ (we have ignored, as customary in the functional integral context, irrelevant factors which in this case are independent of the curve and the field). The $\sqrt{g(\tau)}$ factor has been introduced in order to have reparametrization invariance. In the limit $\lambda \rightarrow \infty$, the mass of the degrees of freedom of the field localized in $\mathcal{C}$ becomes infinite, and therefore the effect of this quadratic term in the action becomes equivalent to that of the $\delta_{\mathcal{C}}(\varphi)$ mentioned in Eq. (2)

$$
\delta_{\mathcal{C}}(\varphi)=\prod_{\tau} \delta(\varphi(y(\tau))) .
$$

It is rather convenient to use an auxiliary field $\xi(\tau)$, in order to have an alternative representation for the functional above, where $\varphi$ may be integrated out in a simpler fashion. Indeed,

$$
\mathcal{Z}_{\lambda}(\mathcal{C})=\int \mathcal{D} \varphi \mathcal{D} \xi e^{-\frac{1}{2} \int_{x}\left(\partial_{\mu} \varphi \partial_{\mu} \varphi+m^{2} \varphi^{2}\right)+i \int_{x} J_{\mathcal{C}} \varphi-\frac{1}{2 \lambda} \int_{\tau} \sqrt{g(\tau)}[\xi(\tau)]^{2}}
$$


where we have dropped a factor corresponding to the integration of $\xi$ in the absence of sources, since it gives rise to a divergence proportional to the mirror's mass. Also, we defined

$$
J_{\mathcal{C}}(x) \equiv \int_{\tau} \sqrt{g(\tau)} \xi(\tau) \delta(x-y(\tau))
$$

Integrating out $\varphi$, we see that

$$
\mathcal{Z}_{\lambda}(\mathcal{C})=\mathcal{Z}_{0} \int \mathcal{D} \xi e^{-\frac{1}{2} \int_{\tau, \tau^{\prime}} \xi(\tau) \mathcal{K}\left(\tau, \tau^{\prime}\right) \xi\left(\tau^{\prime}\right)}
$$

with a kernel $\mathcal{K}$ which may be rendered as follows:

$$
\begin{aligned}
\mathcal{K}\left(\tau, \tau^{\prime}\right)= & \sqrt{g(\tau)}\left[\frac{\delta\left(s(\tau)-s\left(\tau^{\prime}\right)\right)}{\lambda}\right. \\
& \left.+\left\langle y(\tau)\left|\left(-\partial^{2}+m^{2}\right)^{-1}\right| y\left(\tau^{\prime}\right)\right\rangle\right] \sqrt{g\left(\tau^{\prime}\right)},
\end{aligned}
$$

where $s(\tau)$ denotes the Euclidean version of the proper time, namely, the arc length, and we have used a bra-ket notation for the kernel of an operator.

Then, integration of the auxiliary field yields

$$
\mathcal{Z}_{\lambda}(\mathcal{C})=\mathcal{Z}_{0}(\operatorname{det} \mathcal{K})^{-1 / 2},
$$

and finally

$$
\Gamma_{\lambda}(\mathcal{C})=\frac{1}{2} \operatorname{Tr} \log \mathcal{K} .
$$

We proceed in the next section to perform a perturbative expansion of the effective action in powers of the departure of the particle as measured with respect to a static situation.

\section{SMALL-DEPARTURE EXPANSION FOR THE MASSLESS FIELD}

We consider a worldline $\mathcal{C}$ parametrized with the "Lab" time $\tau \equiv t \equiv x_{0}$. Therefore, $\left(y_{\mu}\right)=\left(y_{\mu}(t)\right)=\left(t, \eta_{i}(t)\right)$ $(i=1, \ldots, d)$, and we assume that $\eta_{i}(t)$, the departure from a static situation, $(t, \mathbf{0})$, is small. By an adequate choice of the spatial origin, we can always assume that the average position of the particle is $\mathbf{0}$; thus: $\int_{t} \eta_{i}(t)=0$. Besides, we deal with nonrelativistic motions, so that the $\sqrt{g}$ factors will be replaced by 1 .

We proceed to perform an expansion in powers of $\eta_{i}(t)$. Using an index to denote, in a given object, the order in $\eta_{i}$ in that expansion, we shall have

$$
\Gamma_{\lambda}(\mathcal{C})=\Gamma_{\lambda}^{(0)}(\mathcal{C})+\Gamma_{\lambda}^{(1)}(\mathcal{C})+\Gamma_{\lambda}^{(2)}(\mathcal{C})+\ldots
$$

where the explicit form of the first few terms is

$$
\begin{aligned}
\Gamma_{\lambda}^{(0)}(\mathcal{C})= & \frac{1}{2} \operatorname{Tr} \log \mathcal{K}^{(0)}, \\
\Gamma_{\lambda}^{(1)}(\mathcal{C})= & \frac{1}{2} \operatorname{Tr}\left[\left(\mathcal{K}^{(0)}\right)^{-1} \mathcal{K}^{(1)}\right], \\
\Gamma_{\lambda}^{(2)}(\mathcal{C})= & \frac{1}{2} \operatorname{Tr}\left[\left(\mathcal{K}^{(0)}\right)^{-1} \mathcal{K}^{(2)}\right] \\
& -\frac{1}{4} \operatorname{Tr}\left[\left(\mathcal{K}^{(0)}\right)^{-1} \mathcal{K}^{(1)}\left(\mathcal{K}^{(0)}\right)^{-1} \mathcal{K}^{(1)}\right] .
\end{aligned}
$$

It goes without saying that the $\Gamma_{\lambda}^{(0)}(\mathcal{C})$, independent of the particle's motion, may be safely discarded, and we shall do so (it only contributes a constant to the static vacuum energy).

To evaluate the remaining terms, we need to consider the kernels $\mathcal{K}^{(i)}, i=0,1,2$. We see that

$$
\begin{aligned}
\mathcal{K}_{\lambda}^{(0)}\left(t, t^{\prime}\right) & =\frac{1}{\lambda} \delta\left(t-t^{\prime}\right)+\left\langle t, \mathbf{0}\left|\left(-\partial^{2}\right)^{-1}\right| t^{\prime}, \mathbf{0}\right\rangle \\
& =\int \frac{d \omega}{2 \pi} e^{i \omega\left(t-t^{\prime}\right)} \tilde{\mathcal{K}}_{\lambda}^{(0)}(\omega)
\end{aligned}
$$

where

$$
\tilde{\mathcal{K}}_{\lambda}^{(0)}(\omega)=\frac{1}{\lambda}+I(\omega),
$$

where

$$
I(\omega)=\int \frac{d^{d} k}{(2 \pi)^{d}} \frac{1}{\mathbf{k}^{2}+\omega^{2}}
$$

(we have used $\omega$ to denote the $k_{0}$ component of the momentum).

It is rather straightforward to see that $\mathcal{K}_{\lambda}^{(1)}$ vanishes

$$
\begin{aligned}
\mathcal{K}_{\lambda}^{(1)}\left(t, t^{\prime}\right)= & i \int \frac{d \omega}{2 \pi} e^{i \omega\left(t-t^{\prime}\right)} \\
& \times \int \frac{d^{d} k}{(2 \pi)^{d}} \frac{1}{\mathbf{k}^{2}+\omega^{2}} k_{j}\left(\eta_{j}(t)-\eta_{j}\left(t^{\prime}\right)\right)=0 .
\end{aligned}
$$

Regarding $\mathcal{K}_{\lambda}^{(2)}$, we obtain

$$
\begin{aligned}
\mathcal{K}_{\lambda}^{(2)}\left(t, t^{\prime}\right)= & -\frac{1}{2} \int \frac{d \omega}{2 \pi} e^{i \omega\left(t-t^{\prime}\right)} \int \frac{d^{d} k}{(2 \pi)^{d}} \frac{k_{i} k_{j}}{\mathbf{k}^{2}+\omega^{2}} \\
& \times\left(\eta_{i}(t)-\eta_{i}\left(t^{\prime}\right)\right)\left(\eta_{j}(t)-\eta_{j}\left(t^{\prime}\right)\right) .
\end{aligned}
$$

Or,

$$
\begin{aligned}
\mathcal{K}_{\lambda}^{(2)}\left(t, t^{\prime}\right)= & -\frac{1}{2 d} \int \frac{d \omega}{2 \pi} e^{i \omega\left(t-t^{\prime}\right)} \int \frac{d^{d} k}{(2 \pi)^{d}} \frac{\mathbf{k}^{2}}{\mathbf{k}^{2}+\omega^{2}} \\
& \times\left(\eta_{i}(t)-\eta_{i}\left(t^{\prime}\right)\right)^{2} .
\end{aligned}
$$


We see that

$$
\begin{aligned}
\mathcal{K}_{\lambda}^{(2)}\left(t, t^{\prime}\right)= & \frac{1}{2 d} \int \frac{d \omega}{2 \pi} e^{i \omega\left(t-t^{\prime}\right)} \omega^{2} I(\omega)\left(\eta_{i}(t)-\eta_{i}\left(t^{\prime}\right)\right)^{2} \\
= & \frac{1}{2 d}\left[\left(\eta_{i}(t)\right)^{2}+\left(\eta_{i}\left(t^{\prime}\right)\right)^{2}\right] \int \frac{d \omega}{2 \pi} e^{i \omega\left(t-t^{\prime}\right)} \omega^{2} I(\omega) \\
& -\frac{1}{d} \eta_{i}(t) \eta_{i}\left(t^{\prime}\right) \int \frac{d \omega}{2 \pi} e^{i \omega\left(t-t^{\prime}\right)} \omega^{2} I(\omega)
\end{aligned}
$$

It is evident that the first-order term $\Gamma_{\lambda}^{(1)}$ vanishes. Let us then calculate $\Gamma_{\lambda}^{(2)}$, the only surviving contribution:

$$
\Gamma_{\lambda}^{(2)}=\frac{1}{2} \operatorname{Tr}\left(\Delta \mathcal{K}_{\lambda}^{(2)}\right)
$$

with $\Delta \equiv\left(\mathcal{K}_{\lambda}^{(0)}\right)^{-1}$. Then

$$
\begin{aligned}
\Gamma_{\lambda}^{(2)}= & \frac{1}{2} \int_{t, t^{\prime}} \Delta\left(t, t^{\prime}\right) \mathcal{K}_{\lambda}^{(2)}\left(t^{\prime}, t\right) \\
= & \int \frac{d \omega}{2 \pi}\left[\tilde{\mathcal{K}}_{\lambda}^{(0)}(\omega)\right]^{-1} e^{i \omega\left(t-t^{\prime}\right)} \mathcal{K}_{\lambda}^{(2)}\left(t^{\prime}, t\right) \\
= & \frac{1}{d} \int_{t}\left(\eta_{i}(t)\right)^{2} \int \frac{d \omega}{2 \pi}\left[\tilde{\mathcal{K}}_{\lambda}^{(0)}(\omega)\right]^{-1} \omega^{2} I(\omega) \\
& +\frac{1}{2} \int \frac{d \omega}{2 \pi} f(\omega)\left|\tilde{\eta}_{i}(\omega)\right|^{2}
\end{aligned}
$$

where $\tilde{\eta}_{i}$ is the Fourier transform of $\eta_{i}$, and

$$
f(\omega)=-\frac{1}{d} \int \frac{d \nu}{2 \pi}\left[\tilde{\mathcal{K}}_{\lambda}^{(0)}(\nu+\omega)\right]^{-1} \nu^{2} I(\nu) .
$$

Note that the first term in the second line of (23) is a renormalization in the mirror's mass; we shall focus in what follows on the properties of the second one. The treatment of such a term differs depending on the number $d$ of spatial dimensions. Indeed, we see that $f$ depends on $I(\omega)$, both explicitly and also through $\tilde{\mathcal{K}}_{\lambda}^{(0)}$, and $I(\omega)$ diverges, except for $d=1$. We note that the very same divergence appears when considering the $\delta$-function potential in $d>1$. This requires one to renormalize the coupling $\lambda$, something which we will implement here as well.

One can also see that, since $\mathcal{K}^{(1)}$ vanishes, the expression for the fourth-order term simplifies to

$$
\begin{aligned}
\Gamma_{\lambda}^{(4)}(\mathcal{C})= & \frac{1}{2} \operatorname{Tr}\left[\left(\mathcal{K}^{(0)}\right)^{-1} \mathcal{K}^{(4)}\right] \\
& -\frac{1}{4} \operatorname{Tr}\left[\left(\mathcal{K}^{(0)}\right)^{-1} \mathcal{K}^{(2)}\left(\mathcal{K}^{(0)}\right)^{-1} \mathcal{K}^{(2)}\right] .
\end{aligned}
$$

\section{A. $d=1$}

The $d=1$ case has been previously studied [3]. In this case, no renormalization of $\lambda$ is required, since the integral $I(\omega)$ is convergent. Indeed,

$$
\left.[I(\omega)]\right|_{d=1}=\frac{1}{2|\omega|}
$$

The $\nu$ integral in the expression for $f$ can then be explicitly evaluated, the result being

$f(\omega)=-\frac{\lambda^{2}}{8 \pi}\left[2|\omega|-\lambda\left(1+\frac{2}{\lambda}|\omega|\right) \ln \left(1+\frac{2}{\lambda}|\omega|\right)\right]$.

To obtain this result, the zero frequency contribution had to be subtracted. For details, see Sec. II A. of Ref. [3]. A large$\lambda$ expansion of the previous expression yields

$$
f(\omega)=\frac{\lambda}{4 \pi} \omega^{2}-\frac{1}{6 \pi}|\omega|^{3}+\mathcal{O}\left(\lambda^{-1}\right),
$$

where one observes the different nature of the terms; the second one is the well-known Dirichlet result, and the first one amounts to a renormalization of the kinetic energy of the particle.

\section{B. $d=3$}

In three spatial dimensions, we see that

$$
[I(\omega)]_{d=3}=\frac{\Lambda}{2 \pi^{2}}-\frac{|\omega|}{4 \pi},
$$

where $\Lambda$ is a frequency cutoff. Inserting this into $\tilde{\mathcal{K}}_{\lambda}^{(0)}$, we now obtain instead

$$
\tilde{\mathcal{K}}_{\lambda}^{(0)}(\omega)=\frac{1}{\lambda_{r}}-\frac{|\omega|}{4 \pi},
$$

where

$$
\frac{1}{\lambda_{r}}=\frac{1}{\lambda}+\frac{\Lambda}{2 \pi^{2}}
$$

Let us evaluate the kernel $f(\omega)$ for $d=3$, for the case $\lambda_{r} \rightarrow \infty$. We see that, after dropping divergences proportional to the mass and kinetic energy of the mirror,

$$
f_{D}(\omega) \equiv[f(\omega)]_{\lambda_{r} \rightarrow \infty}=-\frac{1}{3} \int \frac{d \nu}{2 \pi} \frac{|\nu|^{3}}{|\nu+\omega|} .
$$

The last integral may be obtained as

$$
\begin{aligned}
f_{D}(\omega) & =f(\omega ;-3 / 2,1 / 2), \\
\left.f\left(\omega ; \alpha_{1}, \alpha_{2}\right)\right] & =-\frac{1}{3} \int \frac{d \nu}{2 \pi} \frac{1}{\left|\nu^{2}\right|^{\alpha_{1}}\left[(\nu+\omega)^{2}\right]^{\alpha_{2}}},
\end{aligned}
$$

where, after a standard calculation, we find 


$$
\begin{aligned}
& \left.f\left(\omega ; \alpha_{1}, \alpha_{2}\right)\right] \\
& \quad=-\frac{\Gamma\left(\alpha_{1}+\alpha_{2}-1 / 2\right)\left[\Gamma\left(3 / 2-\alpha_{1}-\alpha_{2}\right)\right]^{2}}{3(4 \pi)^{1 / 2} \Gamma\left(\alpha_{1}\right) \Gamma\left(\alpha_{2}\right) \Gamma\left(3-2 \alpha_{1}-2 \alpha_{2}\right)}|\omega|^{1-2\left(\alpha_{1}+\alpha_{2}\right)} .
\end{aligned}
$$

Thus,

$$
f_{D}(\omega)=-\frac{1}{256}|\omega|^{3} .
$$

We can also calculate explicitly the subleading terms. We have found that all the terms involving even powers of the frequency $\omega$, and therefore not contributing to the dissipative effects (imaginary part of the analytically continued $\Gamma$ ), are divergent. The terms which are odd in $|\omega|$, including the $\lambda_{r} \rightarrow \infty$ one, are finite:

$f(\omega)=-\frac{1}{256}|\omega|^{3}+\frac{\pi^{2}}{\lambda_{r}^{2}}|\omega|-\frac{64 \pi^{4}}{3 \lambda_{r}^{4}}|\omega|^{-1}+\mathcal{O}\left(\frac{1}{\lambda_{r}^{6}}\right)$.

Therefore, performing the rotation back to real time,

$$
\begin{aligned}
\operatorname{Im}\left[\Gamma_{\lambda}^{(2)}\right]= & \frac{1}{2} \int \frac{d \omega}{2 \pi}\left|\tilde{\eta}_{i}(\omega)\right|^{2} \\
& \times\left[\frac{1}{256}|\omega|^{3}-\frac{\pi^{2}}{\lambda_{r}^{2}}|\omega|+\frac{64 \pi^{4}}{3 \lambda_{r}^{4}}|\omega|^{-1}-\ldots\right] .
\end{aligned}
$$

\section{THE $d=2$ EFFECTIVE ACTION AND $s$-SPACE RENORMALIZATION}

\section{A. $m=0$}

In $d=2$, we note that the integral in $I(\omega)$ is logarithmically divergent. Introducing an UV cutoff $\Lambda$, we see that, for large values of $\Lambda$,

$$
[I(\omega)]_{d=2}=\frac{1}{2 \pi} \log \left|\frac{\Lambda}{\omega}\right| .
$$

Following the usual treatment of the $\delta$-function potential, we introduce a renormalization scale $\mu$, and rewrite

$$
[I(\omega)]_{d=2}=\frac{1}{2 \pi} \log \left|\frac{\Lambda}{\mu}\right|-\frac{1}{2 \pi} \log \left|\frac{\omega}{\mu}\right| .
$$

Inserting this into $\tilde{\mathcal{K}}_{\lambda}^{(0)}$, we see that

$$
\tilde{\mathcal{K}}_{\lambda}^{(0)}(\omega)=\frac{1}{\lambda_{r}}+I_{\mu}(\omega)
$$

where we have introduced the renormalized coupling constant

$$
\frac{1}{\lambda_{r}}=\frac{1}{\lambda}+\frac{1}{2 \pi} \log \left|\frac{\Lambda}{\mu}\right|,
$$

and the scale-dependent function

$$
I_{\mu}(\omega)=-\frac{1}{2 \pi} \log \left|\frac{\omega}{\mu}\right| .
$$

Note that $I(\omega)$ also appears in the denominator of (24); using (39), one sees that

$$
\begin{aligned}
f(\omega)= & -\frac{1}{4 \pi} \log \left|\frac{\Lambda}{\mu}\right|\left[\int \frac{d \nu}{2 \pi}\left[\tilde{\mathcal{K}}_{\lambda}^{(0)}(\nu)\right]^{-1} \nu^{2}\right. \\
& \left.+\omega^{2} \int \frac{d \nu}{2 \pi}\left[\tilde{\mathcal{K}}_{\lambda}^{(0)}(\nu)\right]^{-1}\right]+f_{\mu}(\omega),
\end{aligned}
$$

where the first two terms amount to renormalizations of the mass and kinectic energy of the mirror, while

$$
f_{\mu}(\omega)=-\frac{1}{2} \int \frac{d \nu}{2 \pi}\left[\frac{1}{\lambda_{r}}+I_{\mu}(\nu+\omega)\right]^{-1} \nu^{2} I_{\mu}(\nu),
$$

which does not involve divergent objects in its integrand.

Let us now evaluate the previous integral, which may be rendered in the following fashion:

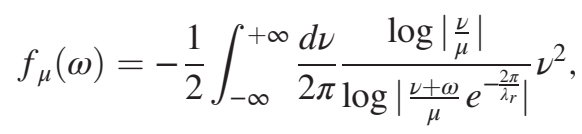

where we have indicated the range of integration explicitly.

To perform the integral, we first perform a shift in the integration variable, and symmetrize it explicitly with respect to $\nu$. Then we obtain the equivalent expression:

$$
\begin{aligned}
f_{\mu}(\omega)= & -\frac{1}{4 \pi} \int_{0}^{\infty} d \nu\left[\frac{\log \left|\frac{\nu+\omega}{\mu}\right|}{\log \mid \frac{\nu}{\mu} e^{-\frac{2 \pi}{\lambda_{r}} \mid}}(\nu+\omega)^{2}\right. \\
& \left.+\frac{\log \left|\frac{\nu-\omega}{\mu}\right|}{\log \mid \frac{\nu}{\mu} e^{-\frac{2 \pi}{\lambda_{r}} \mid}}(\nu-\omega)^{2}\right] .
\end{aligned}
$$

The last integral is UV divergent; to cope with those divergences, we subtract from the integrand its Taylor expansion around $\omega=0$, up to the second order. This procedure does not erase information related to dissipation, as the subtracted terms give rise to a renormalization of the kinetic term and the of the particle. This leads (after some algebra) to the subtracted integral $f_{s}$ :

$$
\begin{aligned}
f_{s}(\omega)= & -\frac{1}{4 \pi} \int_{0}^{\infty} \frac{d \nu}{\log \left|\frac{\nu}{\mu} e^{-\frac{2 \pi}{\lambda_{r}}}\right|}\left[\log \left|1-\left(\frac{\omega}{\nu}\right)^{2}\right|\left(\nu^{2}+\omega^{2}\right)\right. \\
& \left.+2 \log \left|\frac{\nu+\omega}{\nu-\omega}\right| \nu \omega-3 \omega^{2}\right] .
\end{aligned}
$$

The previous integral is UV convergent. Performing a rescaling in the integration variable, 


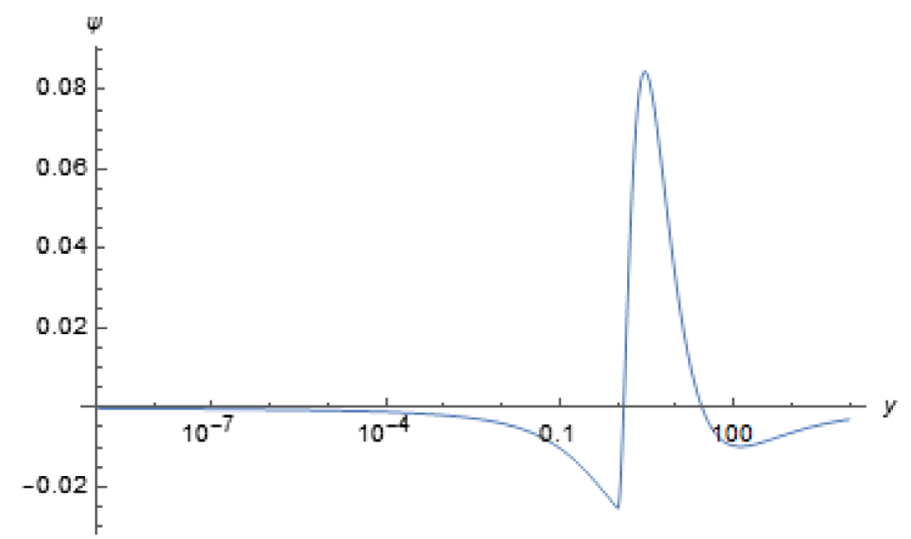

(a)

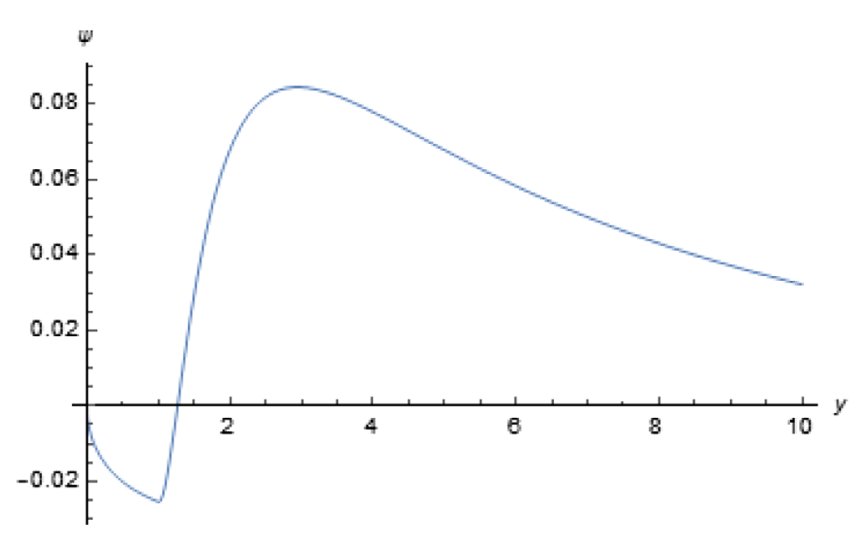

(b)

FIG. 1. (a) $\psi(y)$ as a function of $y \in\left[10^{-9}, 10^{4}\right]$; here we considered a logarithmic (linear) scale for the horizontal (vertical) axis. (b) $\psi(y)$ as a function of $y \in\left[10^{-9}, 10\right]$ (with linear scales).

$$
f_{s}(\omega)=|\omega|^{3} \psi\left(\left|\frac{\omega}{\mu}\right| e^{-\frac{2 \pi}{\lambda_{r}}}\right)
$$

where

$$
\begin{aligned}
\psi(y)= & -\frac{1}{4 \pi} \int_{0}^{\infty} \frac{d x}{\log |x y|}\left[\left(x^{2}+1\right) \log \left|1-\frac{1}{x^{2}}\right|\right. \\
& \left.+2 x \log \left|\frac{x+1}{x-1}\right|-3\right] .
\end{aligned}
$$

$\psi(y)$ may be evaluated numerically, and it turns out to be finite and smooth for every $y>0$, as can be seen in Figs. 1 and 2, which were generated with Mathematica.

As usual, the choice of the subtraction point is parametrized by the arbitrary mass scale $\mu$ which has appeared in the problem. Upon substituting $f(\omega)$ in $(23)$, we see that, for a given $\mu$ and $\lambda_{r}$, there will be a wide range of frequencies receiving a negative contribution to the effective action from this term. More specifically, departures of

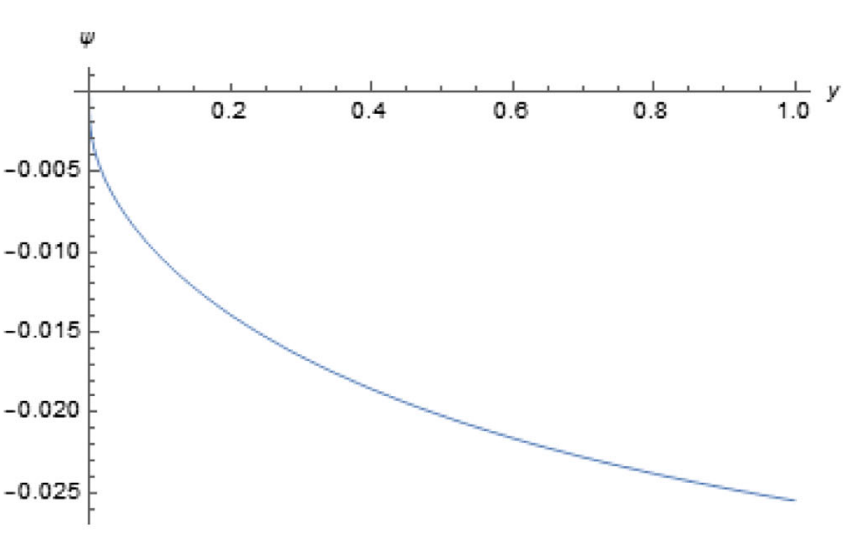

(a) the mirror with frequencies $\omega$ such that $\psi(y)$ is negative will be energetically favored by this term. We have thus succeeded in renormalizing the codimension 2 case in $d=2$. Since $d=2$ exhibits interesting features, let us now consider, for the sake of completeness, also a massive field with mass $m$.

In what follows, we shall consider the arc length $(s)$ of the curve, and introduce a regularization in $s$-parameter space. Our starting point is Eq. (12),

$$
\Gamma_{\lambda}(\mathcal{C})=\frac{1}{2} \operatorname{Tr} \log \left(\hat{1}+\lambda \mathcal{H}_{\mathcal{C}}\right)+\text { const }
$$

where the kernel of the operator $\mathcal{H}_{\mathcal{C}}$, obtained from Eq. (10) as

$\mathcal{H}_{\mathcal{C}}\left(s, s^{\prime}\right)=\left\langle y(s)\left|\left(-\partial^{2}+m^{2}\right)^{-1}\right| y\left(s^{\prime}\right)\right\rangle=\frac{1}{4 \pi} \frac{e^{-m\left|y(s)-y\left(s^{\prime}\right)\right|}}{\left|y(s)-y\left(s^{\prime}\right)\right|}$,

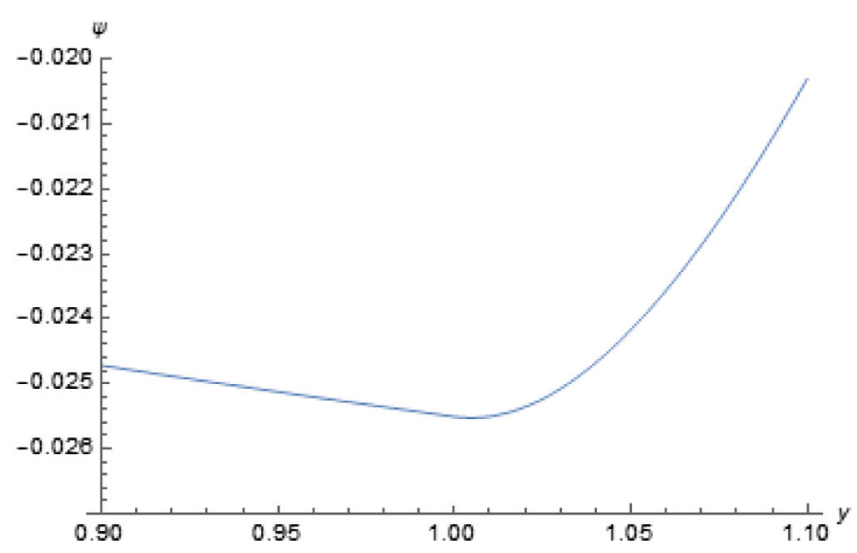

(b)

FIG. 2. (a) Closeup of $\psi(y)$ in the interval $y \in\left[10^{-9}, 1\right]$. (b) Closeup of $\psi(y)$ at the first local minimum of $\psi(y)$, which shows a regular slope. 
is singular when $s \rightarrow s^{\prime}$. This object can be treated by means of a regularization which enables the identification of the singular part in the sense of distributions. For example, we could use dimensional regularization $(d=2-\epsilon)$ and, of course, introduce a mass parameter

$\lambda \rightarrow \lambda \mu^{\epsilon}$ so as to keep $\lambda$ adimensional:

$$
\begin{aligned}
\mathcal{H}_{\mathcal{C}}\left(s, s^{\prime}\right) \rightarrow & \frac{1}{(2 \pi)^{\frac{3-\epsilon}{2}}}\left(\frac{m}{\left|y(s)-y\left(s^{\prime}\right)\right|}\right)^{\frac{1-\epsilon}{2}} \\
& \times K_{\frac{1-\epsilon}{2}}\left(m\left|y(s)-y\left(s^{\prime}\right)\right|\right) .
\end{aligned}
$$

Another possibility is to consider the regularized quantity

$$
\begin{gathered}
\mathcal{H}_{\mathcal{C}}\left(s, s^{\prime}\right) \rightarrow \mathcal{H}_{\mathcal{C}}^{\epsilon}\left(s, s^{\prime}\right)=e^{-m\left|y(s)-y\left(s^{\prime}\right)\right|} \mathcal{I}_{\mathcal{C}}^{\epsilon}\left(s, s^{\prime}\right), \\
\mathcal{I}_{\mathcal{C}}^{\epsilon}\left(s, s^{\prime}\right)=\frac{1}{4 \pi} \frac{\mu^{\epsilon}}{\left|y(s)-y\left(s^{\prime}\right)\right|^{1-\epsilon}} .
\end{gathered}
$$

In order to simplify the ( $s$-independent) finite part, which will be absorbed in the renormalized coupling constant, we shall adopt the second procedure. It is easy to see that in the limit $m \rightarrow 0$ this coincides with the usual dimensional regularization.

\section{Regularization in s-space}

We can initially rewrite

$$
\mathcal{H}_{\mathcal{C}}^{\epsilon}=D^{\epsilon}+\mathcal{H}_{l}^{\epsilon}, \quad D^{\epsilon}\left(s, s^{\prime}\right)=\mathcal{H}_{\mathcal{C}}^{\epsilon}-\mathcal{H}_{l}^{\epsilon}
$$

where $\mathcal{H}_{l}^{\epsilon}$ is the contribution of a line. In fact, the regulator can be removed in $D^{\epsilon}$, as $D=\mathcal{H}_{\mathcal{C}}-\mathcal{H}_{l}$ is regular when $s \rightarrow s^{\prime}$. To see this, we can expand $y\left(s^{\prime}\right)$ around $s$ :

$$
\begin{aligned}
y\left(s^{\prime}\right)-y(s)= & y^{\prime}(s)\left(s^{\prime}-s\right)+\frac{y^{\prime \prime}(s)}{2}\left(s^{\prime}-s\right)^{2} \\
& +\frac{y^{\prime \prime \prime}(s)}{3 !}\left(s^{\prime}-s\right)^{3}+\ldots,
\end{aligned}
$$

and use that for the arc-length parameter it is verified $\left[e(s)=y^{\prime}(s)\right]$

$$
|e(s)|^{2}=1, \quad e(s) \cdot e^{\prime}(s)=0, \quad e(s) \cdot e^{\prime \prime}(s)=-\left|e^{\prime}(s)\right|^{2} .
$$

In other words,

$$
\begin{aligned}
\left|y(s)-y\left(s^{\prime}\right)\right| & =\left|s-s^{\prime}\right|\left(1+h\left(s, s^{\prime}\right)\right), \\
h\left(s, s^{\prime}\right) & =-\frac{\dot{e}^{2}(s)}{24}\left(s-s^{\prime}\right)^{2}+\ldots
\end{aligned}
$$

where the dots represent orders higher than $\left(s-s^{\prime}\right)^{2}$. Therefore,

$$
D\left(s, s^{\prime}\right)=\frac{1}{4 \pi}\left(\frac{e^{-m\left|y(s)-y\left(s^{\prime}\right)\right|}}{\left|y(s)-y\left(s^{\prime}\right)\right|}-\frac{e^{-m\left|s-s^{\prime}\right|}}{\left|s-s^{\prime}\right|}\right)
$$

is manifestly regular when $s$ approaches $s^{\prime}$. In particular

$$
D(s, s)=\lim _{s \rightarrow s^{\prime}} D\left(s, s^{\prime}\right)=0 .
$$

Now, let us analyze

$$
\mathcal{H}_{l}^{\epsilon}\left(s, s^{\prime}\right)=e^{-m\left|s-s^{\prime}\right|} \mathcal{I}_{l}^{\epsilon}\left(s, s^{\prime}\right), \quad \mathcal{I}_{l}^{\epsilon}\left(s, s^{\prime}\right)=\frac{1}{4 \pi} \frac{\mu^{\epsilon}}{\left|s-s^{\prime}\right|^{1-\epsilon}},
$$

which is the product of a regular factor times a distribution with singularities. It is well known that the distribution $|x|^{\alpha}$ has a simple pole at $\alpha=-1$ whose residue is $2 \delta(x)$ [16]. Then, defining the dimensionless variable $x \equiv \mu\left(s-s^{\prime}\right)$, subtracting and adding the polar part of $\mathcal{I}_{l}^{\epsilon}\left(s, s^{\prime}\right)$, and then multiplying by the regular factor $e^{-m\left|s-s^{\prime}\right|}$, we get

$$
\begin{aligned}
\mathcal{H}_{l}^{\epsilon}\left(s, s^{\prime}\right) & =R^{\epsilon}\left(s, s^{\prime}\right)+\frac{1}{2 \pi \epsilon} \delta\left(s-s^{\prime}\right), \\
R^{\epsilon}\left(s, s^{\prime}\right) & =\mathcal{H}_{l}^{\epsilon}\left(s, s^{\prime}\right)-\frac{1}{2 \pi \epsilon} \delta\left(s-s^{\prime}\right) .
\end{aligned}
$$

We can check that $R^{\epsilon}\left(s, s^{\prime}\right)$ is regular when $\epsilon \rightarrow 0$ by acting on a test function

$$
\begin{aligned}
& \int_{-\infty}^{+\infty} d s^{\prime} R^{\epsilon}\left(s, s^{\prime}\right) f\left(s^{\prime}\right) \\
& =\int_{\left|s-s^{\prime}\right| \leq \frac{1}{\mu}} d s^{\prime} \frac{1}{4 \pi} \frac{\mu^{\epsilon}}{\left|s-s^{\prime}\right|^{1-\epsilon}}\left(e^{-m\left|s-s^{\prime}\right|} f\left(s^{\prime}\right)-f(s)\right) \\
& \quad+\int_{\left|s-s^{\prime}\right| \geq \frac{1}{\mu}} d s^{\prime} \frac{1}{4 \pi} \frac{\mu^{\epsilon}}{\left|s-s^{\prime}\right|^{1-\epsilon}} e^{-m\left|s-s^{\prime}\right|} f\left(s^{\prime}\right) .
\end{aligned}
$$

Indeed, this is well defined in the limit $\epsilon \rightarrow 0$. Then, introducing the renormalized coupling constant $\lambda_{r}$,

$$
\frac{1}{\lambda_{r}}=\frac{1}{\lambda}+\frac{1}{2 \pi \epsilon},
$$

the contribution to the effective action is obtained from the $\epsilon \rightarrow 0$ limit of

$$
\Gamma_{\lambda}(\mathcal{C})=\frac{1}{2} \operatorname{Tr} \log \left(\frac{1}{\lambda_{r}}+D+R^{\epsilon}\right)
$$

up to an irrelevant constant.

\section{The weak $\lambda_{r}$ limit}

We may perform an expansion for small $\lambda_{r}$ :

$$
\Gamma_{\lambda}(\mathcal{C})=\delta \Gamma_{\lambda}^{(1)}(\mathcal{C})+\delta \Gamma_{\lambda}^{(2)}(\mathcal{C})+\ldots
$$


The first and second order contributions read, respectively,

$$
\begin{aligned}
\delta \Gamma_{\lambda}^{(1)}(\mathcal{C})= & \frac{\lambda_{r}}{2} \int d s D(s, s)+\frac{\lambda_{r}}{2} \int d s R^{\epsilon}(s, s), \\
\delta \Gamma_{\lambda}^{(2)}(\mathcal{C})= & -\frac{\lambda_{r}^{2}}{4} \int d s d s^{\prime}\left(D^{2}\left(s, s^{\prime}\right)\right. \\
& \left.+2 D\left(s, s^{\prime}\right) R^{\epsilon}\left(s^{\prime}, s\right)+R^{\epsilon 2}\left(s, s^{\prime}\right)\right) .
\end{aligned}
$$

Because of Eq. (60), the first term in Eq. (67) vanishes, while the second term, in spite of the regularization, is still an ill-defined divergent quantity. The same happens with the $R^{2}$ contribution in Eq. (68). However, any improved regularization that keeps the natural dependence of $R$ in $s-s^{\prime}$, which represents the translation symmetry of the contribution along the line, will give a divergence proportional to the length of the curve $\int d s=L \rightarrow \infty$. This is associated with a renormalization of the string tension. In the $\epsilon \rightarrow 0$ limit, the cross term in Eq. (68), integrated over $s^{\prime}$, gives [cf. Eq. (63)]

$$
\begin{aligned}
- & \frac{\lambda_{r}^{2}}{2} \int d s d s^{\prime} R\left(s, s^{\prime}\right) D\left(s, s^{\prime}\right) \\
= & -\frac{\lambda_{r}^{2}}{2} \int_{-\infty}^{\infty} d s \int_{\left|s-s^{\prime}\right| \geq \frac{1}{\mu}} d s^{\prime} \frac{1}{4 \pi} \frac{e^{-m\left|s-s^{\prime}\right|}}{\left|s-s^{\prime}\right|} D\left(s^{\prime}, s\right) \\
& -\frac{\lambda_{r}^{2}}{2} \int_{-\infty}^{\infty} d s \int_{\left|s-s^{\prime}\right| \leq \frac{1}{\mu}} d s^{\prime} \frac{1}{4 \pi} \frac{1}{\left|s-s^{\prime}\right|} \\
& \times\left(e^{-m\left|s-s^{\prime}\right|} D\left(s^{\prime}, s\right)-D(s, s)\right) .
\end{aligned}
$$

This expression is, by construction, regular. Since $D(s, s)=0$, this can be written in a simpler form:

$$
-\frac{\lambda_{r}^{2}}{2} \int d s d s^{\prime} \frac{1}{4 \pi} \frac{e^{-m\left|s-s^{\prime}\right|} D\left(s, s^{\prime}\right)}{\left|s-s^{\prime}\right|} .
$$

Note that $D\left(s, s^{\prime}\right) \geq 0$, so that this is a negative contribution to the effective action, as well as that originated from the $D^{2}$ term.

\section{The small curvature limit}

An interesting physical situation to analyze is when the acceleration of the particle is small, so that, due to the mass gap, it does not radiate. To obtain the lowest order contribution of acceleration, it will be useful to perform an expansion of $\Gamma_{\lambda}(\mathcal{C})$ in powers of $D$, which tends to zero when $\mathcal{C} \rightarrow l$. For this objective, we can rewrite

$$
\begin{aligned}
\Gamma_{\lambda}(\mathcal{C})= & \frac{1}{2} \operatorname{Tr} \log \left(\lambda_{r}^{-1}+R^{\epsilon}\right)+\frac{1}{2} \operatorname{Tr} \log \left(1+\left(\lambda_{r}^{-1}+R^{\epsilon}\right)^{-1} D\right), \\
\Gamma_{\lambda}(\mathcal{C})= & \Gamma_{\lambda}(l)+\frac{1}{2} \operatorname{Tr}\left(\left(\lambda_{r}^{-1}+R^{\epsilon}\right)^{-1} D\right) \\
& -\frac{1}{4} \operatorname{Tr}\left(\left(\lambda_{r}^{-1}+R^{\epsilon}\right)^{-1} D\left(\lambda_{r}^{-1}+R^{\epsilon}\right)^{-1} D\right)+\ldots .
\end{aligned}
$$

Let us analyze the second term, $\frac{1}{2} \operatorname{Tr}\left(\left(\frac{1}{\lambda_{r}}+R^{\epsilon}\right)^{-1} D\right)=\frac{1}{2} \int d s \int d s^{\prime} Q\left(s-s^{\prime}\right) D\left(s, s^{\prime}\right)$

where $Q\left(s-s^{\prime}\right)$ is the kernel of the operator $\left(\lambda_{r}^{-1}+R^{\epsilon}\right)^{-1}$. The term proportional to $\dot{e}^{2}(s)$ can be obtained by using Eq. (58)

$$
\begin{aligned}
\frac{e^{-m\left|y(s)-y\left(s^{\prime}\right)\right|}}{\left|y(s)-y\left(s^{\prime}\right)\right|} & =\frac{e^{-m\left|s-s^{\prime}\right|}}{\left|s-s^{\prime}\right|} \frac{e^{\frac{m}{2 e^{2}}\left|s-s^{\prime}\right|^{3}}}{\left(1-\frac{1}{24} \dot{e}^{2}\left|s-s^{\prime}\right|^{2}\right)}+\ldots \\
& =\frac{e^{-m\left|s-s^{\prime}\right|}}{\left|s-s^{\prime}\right|}+\dot{e}^{2}(s) P\left(s-s^{\prime}\right)+\ldots \\
P\left(s-s^{\prime}\right) & =\frac{1}{24}\left(\left|s-s^{\prime}\right|+m\left|s-s^{\prime}\right|^{2}\right) e^{-m\left|s-s^{\prime}\right|} \\
D\left(s, s^{\prime}\right) & =\frac{\dot{e}^{2}(s)}{4 \pi} P\left(s-s^{\prime}\right), \\
\operatorname{Tr}\left(\left(\lambda_{r}^{-1}+R^{c}\right)^{-1} D\right) & =\int d s \frac{\dot{e}^{2}(s)}{4 \pi} \int d s^{\prime} Q\left(s-s^{\prime}\right) P\left(s-s^{\prime}\right) \\
& =\int d s \frac{\dot{e}^{2}(s)}{4 \pi} \int d u Q(u) P(u)
\end{aligned}
$$

Now, if the kernel of the operator $R^{\epsilon}$ is $R^{\epsilon}\left(s-s^{\prime}\right)$, then, in terms of the Fourier transforms

$P(u)=\int \frac{d \omega}{2 \pi} \tilde{P}(\zeta) e^{i \zeta u}, \quad R^{\epsilon}(u)=\int \frac{d \zeta}{2 \pi} \tilde{R}^{\epsilon}(\zeta) e^{i \zeta u}$,

we have

$$
\int d u Q(u) P(u)=\int \frac{d \zeta}{2 \pi} \frac{\tilde{P}(\zeta)}{\frac{1}{\lambda_{r}}+\tilde{R}^{\epsilon}(\zeta)} .
$$

$P(\omega)$ is found to be

$\int_{-\infty}^{+\infty} d u \frac{1}{24}\left(|u|+m|u|^{2}\right) e^{-m|u|} e^{-i \zeta u}=\frac{3 m^{4}-6 m^{2} \zeta^{2}-\zeta^{4}}{12\left(m^{2}+\zeta^{2}\right)^{3}}$.

The explicit form of $R^{\epsilon}\left(s-s^{\prime}\right)$ is obtained from Eqs. (61), (62)

$$
R^{\epsilon}\left(s-s^{\prime}\right)=\frac{\mu^{\epsilon}}{4 \pi} \frac{e^{-m\left|s-s^{\prime}\right|}}{\left|s-s^{\prime}\right|^{1-\epsilon}}-\frac{1}{2 \pi \epsilon} \delta\left(s-s^{\prime}\right) .
$$

Performing the Fourier transform, using that for $\epsilon>0$ [16]

$$
\begin{aligned}
\mathcal{F}\left(e^{-m|x|}|x|^{\epsilon-1}\right) & =\mathcal{F}\left(e^{-m x} x_{+}^{\epsilon-1}\right)+\mathcal{F}\left(e^{m x} x_{-}^{\epsilon-1}\right) \\
& =i e^{i(\epsilon-1) \frac{\pi}{2}} \Gamma(\epsilon)(-\zeta+i m)^{-\epsilon}+\text { c.c. },
\end{aligned}
$$

we arrive at 


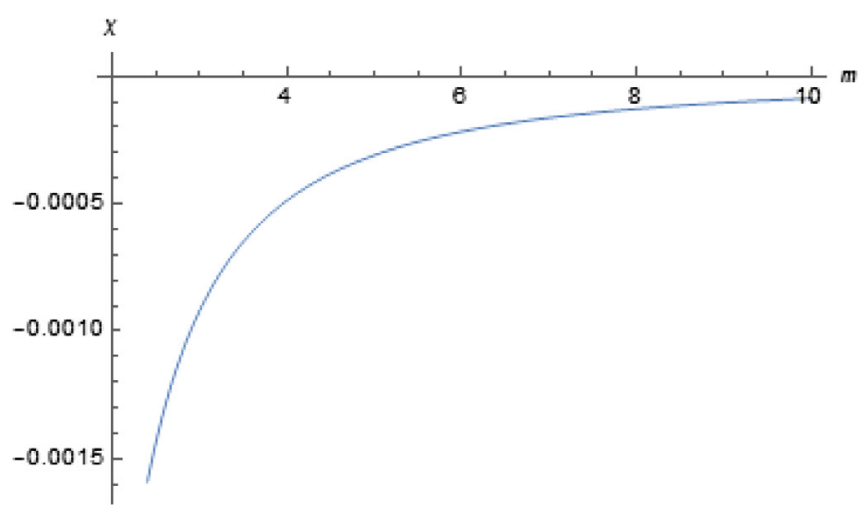

FIG. 3. $\chi$ as a function of $m$, for $\mu=1$ and $\lambda_{r} \rightarrow \infty$.

$\tilde{R}^{\epsilon}(\zeta)=\mathcal{F}\left(R^{\epsilon}\right)(\zeta)=-\frac{\gamma}{2 \pi}-\frac{1}{4 \pi} \log \frac{\left(\zeta^{2}+m^{2}\right)}{\mu^{2}}+O(\epsilon)$.

Here, we can safely take the limit $\epsilon \rightarrow 0$ to conclude

$$
\lim _{\epsilon \rightarrow 0^{+}} \tilde{R}^{\epsilon}(\zeta)=-\frac{\gamma}{2 \pi}-\frac{1}{4 \pi} \log \frac{\left(\zeta^{2}+m^{2}\right)}{\mu^{2}} .
$$

Finally, to get rid of $\gamma$, we redefine $\mu \rightarrow \mu e^{-\gamma}$, which implies

$$
\int d u Q(u) P(u)=\frac{1}{6} \int_{-\infty}^{+\infty} d \zeta \frac{6 m^{2} \zeta^{2}+\zeta^{4}-3 m^{4}}{\left(m^{2}+\zeta^{2}\right)^{3}\left(-\frac{4 \pi}{\lambda_{r}}+\log \frac{\zeta^{2}+m^{2}}{\mu^{2}}\right)} .
$$

Then, to lowest order, we find

$$
\begin{gathered}
\Gamma_{\lambda}(\mathcal{C})-\Gamma_{\lambda}(l)=\chi(m, \mu) \int d s \dot{e}^{2}(s), \\
\chi(m, \mu)=\frac{1}{96 \pi} \int_{-\infty}^{+\infty} d \zeta \frac{6 m^{2} \zeta^{2}+\zeta^{4}-3 m^{4}}{\left(m^{2}+\zeta^{2}\right)^{3} \log \left(\frac{\sqrt{\zeta^{2}+m^{2}}}{\mu} e^{-\frac{2 \pi}{\lambda_{r}}}\right)},
\end{gathered}
$$

where $\Gamma_{\lambda}(l)$ is the effective action of a straight line, and $\chi(m, \mu)$ is a constant that depends on the mass and the arbitrary scale $\mu$. For $m \gg \mu e^{\frac{2 \pi}{\lambda_{r}}}, \chi$ is finite and negative. In Fig. 3, we show the dependence of the $\chi$ coefficient with $m$ in this regime.

\section{CONCLUSIONS}

In this work we have defined and studied quantum dissipation in a moving DCE setting involving $\delta$ interactions with codimension larger than one. Specifically, we studied a real scalar field in $d+1$ dimensions $(d=2$, 3 ) coupled to an imperfect one dimensional mirror. We found that the singular nature of the problem requires a renormalization of the coupling $(\lambda)$ between the field and the mirror. In particular, for $d=3$, there is a finite scaleindependent imaginary part for the effective action. In the Dirichlet limit, similarly to the well-known $d=1$ result, this imaginary part contains a $|\omega|^{3}$ dependence, which is what one expects on dimensional grounds, assuming no renormalization scale dependence is generated.

The case $d=2$, where $\lambda$ is dimensionless, is special, for the coupling not only gets renormalized but also acquires a dependence on a mass scale $\mu$. This phenomenon parallels that observed when dealing with the $\delta$ potential in quantum mechanics for a planar system [8]. In this case, we have shown how the mass scale $\mu$ is generated for the massless field, and how it intervenes in the construction of the renormalized effective action, in the small-departure approximation. We have also found that, for a massive field in $d=2$, apart from inducing renormalizations for the kinetic energy and the mass of the mirror, the Euclidean effective action is finite and negative for small $\lambda_{r}$. Finally, we considered a massive field coupled to an imperfect mirror with small acceleration, where no imaginary part is expected, and found that the effective action is lower than that of a static mirror.

It would be very interesting to extend this analysis to other cases with codimension 2 that arise when considering quantum fluctuations around vortexlike defects in three and four Euclidean dimensions. In particular, it would be important to study the singular problem associated with curved thin center vortices in Yang-Mills theories, and obtain quantum properties such as stiffness from a fundamental point of view.

\section{ACKNOWLEDGMENTS}

We thank Sebastián Franchino-Viñas for pinpointing a missing factor in Eq. (7). The Conselho Nacional de Desenvolvimento Científico e Tecnológico (CNPq), ANPCyT, CONICET, and UNCuyo are acknowledged for financial support. 
[1] G. T. Moore, J. Math. Phys. (N.Y.) 11, 2679 (1970); S. A. Fulling and P. C. W. Davies, Proc. R. Soc. A 348, 393 (1976).

[2] For recent reviews see J. Opt. B 7, S1 (2005); V. V. Dodonov, Phys. Scr. 82 (2010) 038105; D. A. R. Dalvit, P. A. Maia Neto, and F. D. Mazzitelli, Lect. Notes Phys. 834 (2011) 419.

[3] C. D. Fosco, F. C. Lombardo, and F. D. Mazzitelli, Phys. Rev. D 76, 085007 (2007).

[4] C. D. Fosco, F. C. Lombardo, and F. D. Mazzitelli, Phys. Rev. D 84, 025011 (2011).

[5] R. Golestanian and M. Kardar, Phys. Rev. A 58, 1713 (1998).

[6] F. A. Berezin and L. D. Faddeev, Dokl. Akad. Nauk Ser. Fiz. 137, 1011 (1961) [Sov. Math. Dokl. 2, 372 (1961)].

[7] S. Albeverio, R. Hoegh-Krohn, and L. Streit, J. Math. Phys. (N.Y.) 18, 907 (1977).
[8] R. Jackiw, in Diverse Topics in Theoretical and Mathematical Physics (World Scientific, Cambridge, MA, 1995), pp. 35-53.

[9] H. E. Camblong, L. N. Epele, H. Fanchiotti, and C. A. Garcia Canal, Ann. Phys. (Amsterdam) 287, 14 (2001).

[10] R. Golestanian, Phys. Rev. E 62, 5242 (2000).

[11] N. K. Nielsen and P. Olesen, Nucl. Phys. B144, 376 (1978).

[12] H. B. Nielsen and M. Ninomiya, Nucl. Phys. B156, 1 (1979); N. K. Nielsen and P. Olesen, Nucl. Phys. B160, 380 (1979).

[13] H. Leutwyler, Nucl. Phys. B179, 129 (1981); Phys. Lett. B 96, 154 (1980); C. Rajiadakos, Phys. Lett. B 100, 471 (1981).

[14] M. Bordag, Phys. Rev. D 67, 065001 (2003).

[15] L. E. Oxman, J. High Energy Phys. 78 (2011) 1107.

[16] M. Gel'fand and G. E. Shilov, Generalized Functions. Properties and Operations, Vol. 1 (Academic Press, New York, 1964). 